\title{
Towards the Control and Prevention of Waste in IT Service Operation Using Fuzzy Logic: Focus in Incident Management Process
}

\author{
Wadie Berrahal, Rabia Marghoubi and Zineb El Akkaoui \\ National postal and telecommunication institute, Rabat, Morocco
}

\begin{abstract}
All production lines are continuously confronted with the phenomenon of waste, especially in IT operations. A waste is assessed in terms of the required resources and the cost employed to solve the problem behind it. Eliminating the waste in daily operations is essential to improve IT service management. This article aims to provide an estimation of the level of potential waste, where waste generation trends are provoked by the activities of IT service management processes. We are going to focus particularly on the possibility of applying a Lean improvement process to IT services processes when using fuzzy logic method. We specifically demonstrate our contribution through the application of fuzzy analysis to the incident management process. This approach also aims at developing a theoretical and pragmatic model and promoting the knowledge of IT experts. In order to make our framework as generic as possible, concepts of IT operations, including the incident management, are inspired by the Information Technology Infrastructure Library (ITIL), the most prominent framework for IT service governance according to the current literature.
\end{abstract}

ACM CCS (2012) Classification: Software and its engineering $\rightarrow$ Software creation and management $\rightarrow$ Software verification and validation $\rightarrow$ Operational analysis

General and reference $\rightarrow$ Cross-computing tools and techniques $\rightarrow$ Performance

Information systems $\rightarrow$ Information systems applications $\rightarrow$ Process control systems

Keywords: waste management, Lean IT, fuzzy logic, IT operation, incident management

\section{Introduction}

Thinking deeply about purpose, process and, most importantly, focusing on how people achieved their work is the core idea of the Lean philosophy [21]. The Lean methods were born in the industry and the various Lean Assessment Tools (LATs) have been experimented in the engineering industry. The ultimate objective of these methods is to provide perfect value to the customer through value creation process with zero waste. However, despite the fact that wastes negatively impact business, they still persist in organization processes and IT operations. Applying Lean to IT aims to identify and eliminate the sources of lost productivity and, in particular, IT waste during service IT operation. The Lean management recommendations involve both preventive and curative approaches to tackle the waste phenomenon. In practice, preventive solutions enable important optimization [6]. It is now assumed that the success of any IT implementation is especially influenced by its effectiveness in identifying and processing the problems early on, in order to minimize negative impacts on the IT services [24]. This is particularly important as any variation on the IT processes has an immediate impact on customers [19]. On the other hand, it is commonly known that a key factor that guarantees continuous organization improvement is a powerful IT processes management [1]. 
In this paper, we propose a preventive waste approach for any IT operation. We will illustrate our approach through the IT incident management process according to Lean recommendations, and then show how it could be strengthened to other operations. In order to be able to analyze waste, it is required to lay out a convenient design directives for IT operations. We have chosen ITIL [9] as a Lean application environment since it has proven to be the most committed to IT service management [8]. Additionally, the success of ITIL implementation process depends not only on organization artifacts specifications, but also on the pertinence and the consistency of principles provided according to IT service life cycle. As stated by ITIL, any IT operation is managed according to one of the following phases: a) Service Operation (SO), which includes guidance on how to achieve effectiveness and efficiency in the delivery and support of services to ensure value for the client and service provider [3], (b) Continual Service Improvement (CSI), which provides essential guidance to create and maintain value for clients through better conceptualization to operationalization of services [16].

This paper provides a novel approach for preventing waste in IT operations. In order to achieve these results, we proceeded by indepth approach, from abstract to concrete, wich means towards a bottom-up method. Firstly, we defined the scope of intervention for IT service management with the proposal of the model which links type of waste MUDA within the service operation [10]. The second step consists of determining the IT operation management, and consequently fusioning the IT processes and the Lean approach with a new system that integrates the waste as an integral part of the value chain of IT service operation [25]. The third step has as its purpose identification and categorization of the different wastes and their root causes, in order to optimize the existing processes, specially ITIL processes, and enable identifying the weak spots and the activities generating wastes [26]. All this work will allow us the necessary knowledge flow into our control system and estimation based on fuzzy logic method. It is mainly able to identify and evaluate the wastes. As a matter of fact, the aim of applying the fuzzy logic in the incident management is to distinguish several catego- ries of incidents - according to established criteria - with distinct uncertainty and therefore, distinct generated wastes. The delivered output allows IT managers to suitably monitor the required improvement of the IT operations in question. The outline of this work is structured as follows. Section 2 reviews the fuzzy logic applications answering the waste issue in various contexts. Section 3 shows the subtlety of using the fuzzy method in IT operation incident to prevent wastes. Section 4 depicts some fuzzy logic concepts. Section 5 presents our method of prevention and estimation of wastes. The strengths and disadvantages of the method will be discussed in Section 6, with the focus on the case study applied to the incident management process in the context of IT operations. Finally, Section 7 presents the conclusion and perspectives.

\section{Related Work}

\subsection{IT Incident Management}

IT operations are the daily operations handling the management of an organization's information system. In this context, several incidents that might result from service requests are reported with various pertinence levels. For instance, incident management is the process of restoring normal operation when an incident is defined as an interruption of the normal functioning or a reduction in the quality of service [14]. The aim of this operation is to restore service as quickly as possible, using standard resolution processes [12]. Typically, the life cycle of the incident management process is impacted by the priority entry criteria of the incident. It also generally requires specific activities perturbing the process, which generates potential waste. The incident management process is actually not run as a linear flow since it carries out both external and internal flows, e.g., unnecessary movement of material and information, excess work-in-process such as queues and pending requests, unnecessary movement of people in service areas with a poor layout [15] [8]. In addition, the escalation procedure is also known as an important source of delays. 


\subsection{Fuzzy Logic Applications}

Fuzzy methods have been implemented in many areas of research and application such as economics, industry, manufacturing operations, production management, engineering, health sciences, natural sciences, mathematics, services, and information and communication technologies [12]. The fuzzy logic method was developed to use both quantitative and qualitative measures of Lean implementation. In other words, the fuzzy logic allows manipulating both measurable and objective results, and user perceptions [5]. In this sense, an assessment of evaluation methods, including Analytically Hierarchy Process (AHP) and Work In Progress (WIP), were conducted [7]. This study demonstrates that the fuzzy logic method offers several possibilities for analysis by quantitative and qualitative measures. Another comparison work between the Hidden Markov Model (HMM) and fuzzy logic has shown better accuracy for fuzzy logic in assessing web service reliability [11]. In the same way, another study argues the usefulness of fuzzy logic in the quantification of wireless and mobile networks to improve service quality [17]. The fuzzy logic method is used in different research areas in order to make predictions or estimations accurately. Furthermore, performing imagery work using fuzzy methods and generic algorithms optimizes not only the results, but also the parameters of the method of fuzzification [32]. This optimization includes a fusion between several methods, namely particle swarm optimization (PSO) or ant colony optimization (ACO) to enhance the performance of network-based fuzzy inference system ANFIS [30] [31].

Our approach represents one iteration towards a process of continuous improvement in order to better manage the subjectivity, which is represented by fuzzy rules concentrated on the experts of the IT domain. In addition, this approach supports several Lean approaches as much as to improve the management processes in operation and remove waste according to Fuzzy-AHP method [27] [28], but it differs from the fact that it adapts specific altitudes of IT services treatments, namely the intangibility, subjectivity and user perception. This way opens perspectives towards other techniques using machine learning [33] or Fuzzy c-means (FCM) [29].

\section{Preliminary}

As mentioned before, fuzzy methods are frequently used to improve the efficiency of IT operations, as well as for manufacturing operations. In this work, our main purpose is to propose a systematic approach to eliminate all non-value-added activities (waste), which can be associated with activities such as excessive movement, waiting, defects, inappropriate treatment, excessive stocks, transport, lack of knowledge, and overproduction [12].

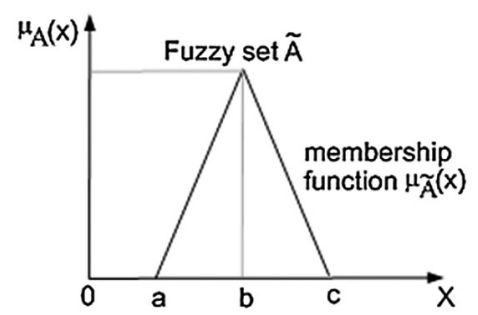

Figure 1. Triangular membership function (Klir and Yuan).

We choose the triangular membership representation depicted in Figure 1. The triangular function is defined by lower limit $a$, an upper limit $c$, and input value $x$, where $a \leq x \leq c$. The $x$-axis represents the universe of discourse of the $x$ criterion, while the $y$-axis represents the degree of belonging in the interval $[a, c]$. The reason for this choice is the fact that the triangular and trapezoidal fuzzy sets are the most common types of fuzzy set operations, due to their simplicity and sufficiency in most analyses [7]. Two ranges of value in this triangular representation could be identified from Figure 1. The first is fuzzified into the input value lower $b$, where $b$ represents midpoint of the opposite side of vertex. The second is fuzzified into the input value upper $b$. The membership function and its different values are readily calculated by using the linear approximation:

$$
\mu(x)=\left\{\begin{array}{cc}
0, & x \leq a \\
\frac{x-a}{b-a}, & a \leq x \leq b \\
\frac{c-x}{c-b}, & b \leq x \leq c \\
0, & x \geq b .
\end{array}\right.
$$


After the application of the fuzzification process of all the inputs, we want the result to be a net value and not a fuzzy set. We therefore need to transform the fuzzy set into a single numerical value as a net worth of outputs. This transformation is performed by using both aggregation and defuzzification methods. The Hamachersum operator is applied as the aggregation method. This choice stems from the fact that the Hamachersum is an operator that seems to model human decision making better than traditional aggregation AND, OR, and union operators because of its compensatory properties and better suitability to empirical data [22]. Besides, although many defuzzification methods have been described in literature, they have generally been applied using standard centroid methods [21]. It is also assumed that the centroid and ensemble maximization and minimization methods are two approaches commonly used for defuzzification [20]. However, as the centroid method is based on the computation of a center of gravity of the aggregated surface, it is more robust to extreme values and could overcome the sensitivity problem in other methods. This prompts its application in our approach.

\section{Systematic Approach of Conttrolling Wastes}

Our approach means to employ the fuzzy logic method in an IT context, particularly in the processes of service operation. In this article, we will experiment with the incident management process to prevent potential waste. Figure 2 . shows the systematic approach proposed. Through its five phases, its final aim is to estimate the degree of wastes that can be generated by treatment of incidents.

In order to be able to perform these phases, we need to prepare the following three main settings:

(i) selection criteria to characterize the input incidents,

(ii) definition of rules of the fuzzy method, and

(iii) evaluation of generated waste.

In other words, our waste prediction model is mainly based on the following three central elements:
- incident criteria: define a set of attributes that characterize a given incident. A criterion is defined as $C_{i}$ qualifying the incident $k$. For a given incident $k, C_{i}$ is evaluated by Value $_{i k}$, where Value $_{i k} \in\left\{l_{i 1}, l_{i 2}, \ldots, l_{i n}\right\}$ and $n \geq 2$ is the number of identified linguistic variables for the criterion $C_{i}$. Linguistic variables are used to quantify the criterion value. Each criterion takes more than one level $l_{i k}$ and constitutes an input for the membership function. Moreover, we define $C=\left\{C_{i} \mid 0 \leq i \leq n\right\}$ as the set of defined criteria. In the present contribution, we considered three criteria: severity, urgency, and gravity.

- generated wastes: represent the evaluation of wastes resulted from a given input of incident criteria. For a given incident $k$, a waste is evaluated by Value $_{k}$, where Value $_{k} \in\left\{d_{1}, d_{2}, \ldots, d_{m}\right\}$ and $m \leq 2$ is the number of linguistic variables for the waste $W_{i}$. Each linguistic variable $d_{k}$ quantifies the degree of the estimated waste according to a given incident. The generated waste is produced by applying respectively the defuzzification and aggregation methods, e.g., centroid and Hamachersum methods, on the fuzzy rules output results. It is worth mentioning that the quantification of the generated waste depends closely of the design of fuzzy rules.

- fuzzy rules: uses fuzzy input values to trigger inference rules and generate fuzzy output values. For the IT incident $k$, the set of rules are defined as:

$$
\begin{gathered}
R_{k}: \text { If }_{1}=\text { Value }_{1 k}[\text { And } / \text { Or }] \ldots C_{n}=\text { Value }_{n k} \\
{\text { Then } W_{k}=\text { Value }_{k}}
\end{gathered}
$$

where, for a given incident $k$, for each $i$, $C_{i}$ represents an input criterion evaluated by Value $_{i k}$ and $W_{k}$ represents the degree of potential waste evaluated by Value $_{k}$. The goal of the established rules is to define the mapping between the set of evaluated criteria and the quantified estimated waste. These rules are written by team IT experts in order to reflect human subjectivity. 


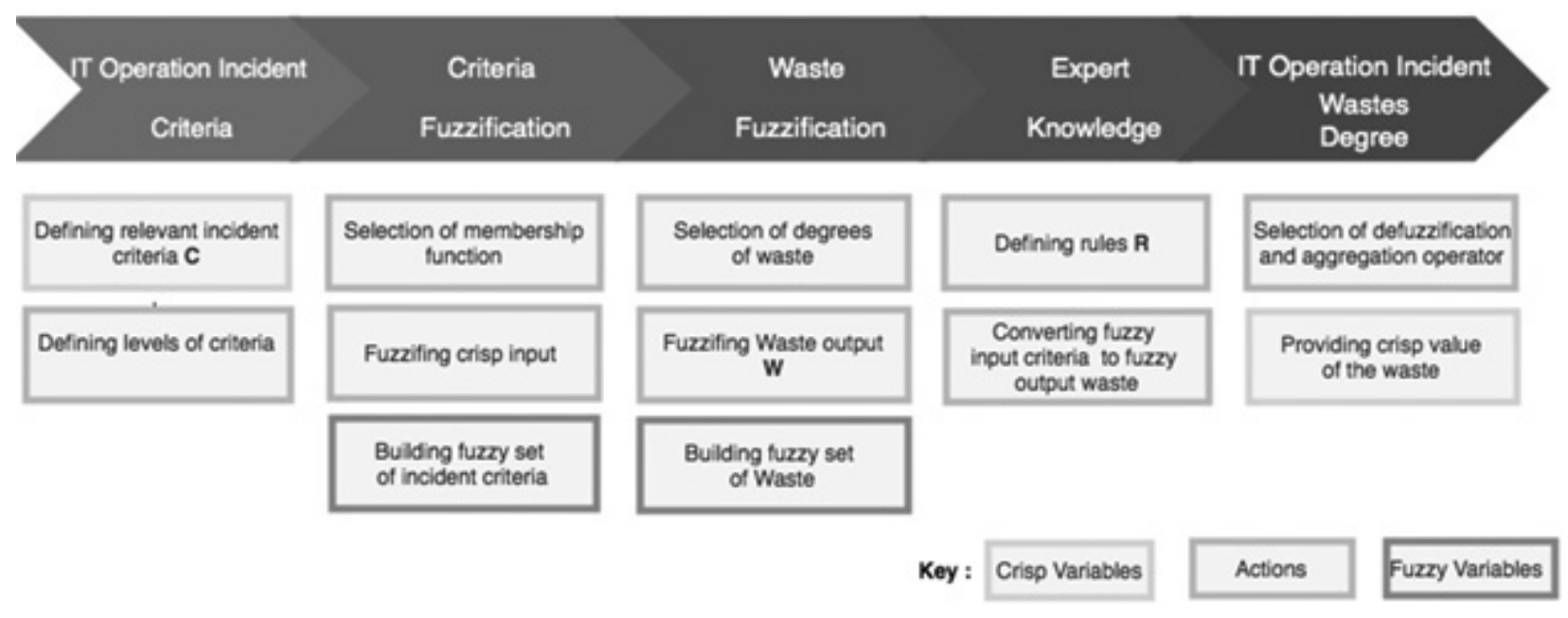

Figure 2. A systematic approach to controlling wastes in IT Incident Management.

\subsection{IT Operation Incident Criteria}

In the following passage, we will exhibit different phases of implementation of the systematic approach for managing IT incidents. The first phase focuses on identifying the incident criteria, all based on ITIL practices[16]. In this context we consider the following criteria:

$$
C_{1}=\text { Severity, } C_{2}=\text { Urgency, } C_{3}=\text { Gravity } .
$$

Table 1. Incident linguistic variable.

\begin{tabular}{|c|c|c|c|c|}
\hline Criteria/Values & 1 & 2 & 3 & 4 \\
\hline Severity & $\overline{\text { Critical }}$ & Major & Minor & Trivial \\
\hline Urgency & Immediate & High & Medium & \\
\hline Gravity & $\begin{array}{l}\text { Blocker } \\
\text { issue }\end{array}$ & $\begin{array}{c}\text { Not } \\
\text { blocker } \\
\text { issue }\end{array}$ & & \\
\hline
\end{tabular}

- Severity: refers to how serious the problem is from an infrastructure perspective and system.

- Urgency: measures how long it will be until an incident has a significant impact on the IT service.

- Gravity: evaluates the effect of an incident on operational processes. Gravity is often based on how deeply the incident problem has affected the service.

\subsection{Criteria Fuzzification}

This phase involves transforming the input criteria linguistic variables, expressed in natural language, into values in the range $[a, b]$, which are fuzzy values. The value is the net minimum value of the criterion and $b$ is its maximum. The fuzzy values are measured by membership functions where $\mu(x)$ is the degree of certainty of the value $x$. The degree relates to membership values where it is between 0 and 1 , since $\mu(x) \in[0,1]$. In the same way, we will exploit our fuzzy set in order to have a waste output in the form of a membership function.

The ultimate purpose of this stage is to build the fuzzy set according to a given incident. This step allows preparing the defuzzification and aggregation step. The fuzzy set is identified as $\left(X=x / Y=d_{j}\right)$, where $x$ is a crisp value for the given incident waste and $d_{j}$ are the degrees that are identified to quantify the waste. In our setting, the fuzzy set for the output variable $Z_{j}$ is defined as the membership function through the triangular representation depicted in Figure 1 . Thus, for each crisp value $Z_{j}$, the waste corresponds to a fuzzy set with a membership function under the following presentation.

Table 2. Waste linguistic variable.

\begin{tabular}{|c||c|c|c|}
\hline Criteria/Value & $\mathbf{1}$ & $\mathbf{2}$ & $\mathbf{3}$ \\
\hline \hline Waste & High & Moderate & Low \\
\hline
\end{tabular}


We also establish the range of the linguistic output variable (Wastes), in Table 2, required for designing the fuzzy sets of a given incident.

Fuzzy set representation of waste:

$$
\mu\left(z_{j}\right)=\frac{\text { value }_{1}}{d_{j 1}}+\frac{\text { value }_{2}}{d_{j 2}}+\frac{\text { value }_{3}}{d_{j 3}},
$$

where $d_{j 1}=$ High, $d_{j 2}=$ Medium, $d_{j 3}=$ Low

\subsection{Expert Knowledge}

At this stage, we have specified both inputs and outputs of the fuzzy sets. Next, we will show how we build and apply the inference rules derived from the technical and functional expertise of the practitioners. These rules represent the knowledge base for the evaluation of potential wastes in the incident management. We use the input values of the criteria to build the inference rules and generate fuzzy output wastes. The structure of the rule conditions is as shown in Table 3.

Table 3. Example of rules.

\begin{tabular}{|l||l|}
\hline 1 & \multicolumn{1}{|c|}{ Expert Rules } \\
\hline \hline $\mathbf{2}$ & $\begin{array}{l}\text { if Severity is Critical and Gravity is Blockerissue } \\
\text { and Urgency is Immediate, then Waste is High }\end{array}$ \\
\hline $\mathbf{3}$ & $\begin{array}{l}\text { if Severity is Critical and Gravity is Blockerissue } \\
\text { and Urgency is High, then Waste is High }\end{array}$ \\
\hline $\mathbf{4}$ & $\begin{array}{l}\text { if Severity is Critical and Gravity is not blockeris- } \\
\text { sue and Urgency is High then Waste is high }\end{array}$ \\
\hline $\mathbf{5}$ & $\begin{array}{l}\text { if Severity is Critical and Gravity is not blockerissue } \\
\text { and Urgency is Medium, then Waste is Moderate }\end{array}$ \\
\hline $\mathbf{5}$ & $\begin{array}{l}\text { if Severity is Major and Gravity is Blockerissue and } \\
\text { Urgency is High, then Waste is High }\end{array}$ \\
\hline $\mathbf{6}$ & $\begin{array}{l}\text { if Severity is Major and Gravity is Blockerissue and } \\
\text { Urgency is Medium, then Waste is Moderate }\end{array}$ \\
\hline $\mathbf{7}$ & $\begin{array}{l}\text { if Severity is Major and Gravity is not blockerissue } \\
\text { and Urgency is High, then Waste is Moderate }\end{array}$ \\
\hline $\mathbf{8}$ & $\begin{array}{l}\text { if Severity is Major and Gravity is not blockerissue } \\
\text { and Urgency is Medium, then Waste is Moderate }\end{array}$ \\
\hline
\end{tabular}

Table 4 gives for each set of incident criteria $C_{i}$ an evaluation by using linguistic variables. Accordingly, the expert evaluates the waste $W_{j}$ by using the corresponding degree $d_{j}$. The defined rules are mainly exploited for estimating the level of generated waste for an input incident.

\subsection{IT Operation Wastes Degree}

In the case of a Mamdani Fuzzy Language Control, the fuzzy accumulation operator is used to accumulate the obtained terms of each output variable to obtain one fuzzy set. The last phase consists of transforming fuzzy values into real values (crisp). For this purpose, we will use the centroid defuzzification method, as justified earlier, to quantify the generated waste. It consists of choosing fuzzy inference methods, i.e. the definition of the defuzzification method, thus converting the fuzzy outputs of each variable into net values. The most commonly used method in this conversion is the centroid defuzzification controller, which is employed to calculate the net value of the surface centroid of the fuzzy set [23]. The completion of this step with a numerical output representing the degree of waste generated by an operational processing of the incident gives an idea of the possible deviations to avoid when managing this process.

\section{Case Study: Incident Management Process}

The case study was conducted in the context of an IT service organization. The incident management process is launched by the user in order to be analyzed and corrected by the technical teams. In this context, we aim at showing how the present approach allows analyzing the wastes generated by the incident management process so as to ensure its continuous improvement. In order to quantify the effect of incidents in terms of waste, we show next how we can reuse the settings described in the previous Section 3.2 .

\subsection{Deployment Context}

The IT service company concerned by the case study is operating in more than sixteen locations across Europe. This leads to multiple technical, language, time zone and regulatory requirements, and thus poses problems for clients. Specifically, the IT environment of the French site includes hardware and software components (servers, operating systems, back office and front office applications, and integra- 
Table 4. Input criteria as regards waste generated.

\begin{tabular}{|c|c|c|c|c|c|c|c|c|c|}
\hline \multirow{3}{*}{ Incident } & \multicolumn{3}{|c|}{ Input Criteria } & \multicolumn{6}{|c|}{ Output Waste } \\
\hline & \multirow{2}{*}{ Severity } & \multirow{2}{*}{ Gravity } & \multirow{2}{*}{ Urgency } & \multicolumn{3}{|c|}{ Waste fuzzy set } & \multirow{2}{*}{$\begin{array}{c}\begin{array}{c}\text { Defuzzified } \\
\text { wastes }\end{array} \\
\begin{array}{c}\text { Centroid of } \\
\text { waste area Y }\end{array}\end{array}$} & \multicolumn{2}{|c|}{ Waste degree } \\
\hline & & & & High & Moderate & Low & & $\begin{array}{c}\text { Estimated } \\
\text { waste }\end{array}$ & $\begin{array}{c}\text { Observed } \\
\text { waste }\end{array}$ \\
\hline INC1 & 1 & 1 & 2 & 0.188 & 0.718 & 0.000 & 1.218 & Moderate & High \\
\hline INC2 & 1 & 2 & 1 & 0.180 & 0.730 & 0.000 & 1.230 & Moderate & High \\
\hline INC3 & 3 & 2 & 3 & 0.000 & 0.088 & 0.608 & 2.412 & Low & Moderate \\
\hline INC4 & 3 & 2 & 2 & 0.000 & 0.556 & 0.296 & 1.944 & Low & Moderate \\
\hline INC5 & 2 & 1 & 3 & 0.000 & 0.796 & 0.136 & 1.704 & Moderate & Moderate \\
\hline INC6 & 2 & 2 & 3 & 0.059 & 0.912 & 0.000 & 1.412 & Moderate & High \\
\hline INC7 & 3 & 2 & 2 & 0.000 & 0.697 & 0.202 & 1.803 & Low & Moderate \\
\hline INC8 & 1 & 1 & 1 & 0.403 & 0.396 & 0.000 & 0.896 & High & High \\
\hline INC10 & 2 & 1 & 2 & 0.235 & 0.647 & 0.000 & 1.147 & Moderate & High \\
\hline INC11 & 4 & 2 & 2 & 0.000 & 0.797 & 0.135 & 1.703 & Low & Moderate \\
\hline INC12 & 4 & 2 & 3 & 0.000 & 0.775 & 0.150 & 1.725 & Low & Moderate \\
\hline INC13 & 4 & 2 & 3 & 0.000 & 0.689 & 0.207 & 1.811 & Low & Moderate \\
\hline INC14 & 2 & 1 & 1 & 0.389 & 0.412 & 0.000 & 0.916 & Moderate & Moderate \\
\hline INC15 & 2 & 1 & 2 & 0.114 & 0.829 & 0.000 & 1.329 & Moderate & High \\
\hline INC16 & 4 & 2 & 2 & 0.000 & 0.092 & 0.605 & 2.408 & Low & Moderate \\
\hline INC17 & 4 & 2 & 3 & 0.000 & 0.039 & 0.641 & 2.461 & Low & Low \\
\hline INC18 & 4 & 2 & 2 & 0.000 & 0.977 & 0.016 & 1.523 & Low & High \\
\hline INC19 & 1 & 1 & 1 & 0.640 & 0.040 & 0.000 & 0.540 & High & High \\
\hline INC20 & 3 & 1 & 2 & 0.000 & 0.700 & 0.200 & 1.800 & Moderate & High \\
\hline
\end{tabular}

tion applications). Applications exchanges are managed centrally, in batches, or through web service. Any type of batch interface uses transfer tables for data exchanging and transforming.

With respect to ITIL standards, the company's IT incident management life cycle is conducted through the following steps. First, we proceed to the identification of the incident where we classify and diagnose the incident. This step allows us to prioritize handling actions. Second, the incident verification step is performed and a report is examined to confirm if it concerns a real issue that deserves attention. If so, the incident resolution cycle is launched. Once the incident resolution is accomplished, the involved parties are informed. Finally, the process is closed. It is noteworthy that incident processing and delivery are managed by releases. For each release, the number of incidents that will be processed and delivered is known in advance. However, there are always exceptions that lead to a revision of the scope and priority of the incident.

\subsection{Fuzzy Logic Approach Deployment}

In this section, we provide a proof of concept of the current approach through its application in a real-world context. The first step consists of building a fuzzy set through input criteria crisp values characterizing each of the reported incidents. 
In the context of IT incident management, the sets of reported incidents are multiple. We focus on a release comprising new incidents about a production line. In our experiments, we have followed the five phases indicated in the systematic approach, see Figure 2. We deployed the steps using a tool called fuzzyLitte that allows implementing and executing the fuzzy steps using Fuzzy Control Language (FCL). Figure 3 represents the execution flow from the input incident criteria to the waste estimation. The execution flow uses the settings described in Section 5.

Through an illustrative example, Figure 3 highlights the relationship between an input incident and its estimated waste. We should start with defining and establishing a set of input criteria and thus fuzzify them using the "triangular membership function". For this example the Severity $=1 \Rightarrow \mu(x)=0.329 /$ Critical $+0.543 /$ Major $+0.000 /$ Minor $+0.000 /$ Trivial Figure 3-a). Then, after applying the fuzzy rules represented as the following form: $R_{k}: S_{i} C_{1}=$ Value $_{1 k}[$ AND/OR $] \ldots C_{n}=$ Value $_{n k}$ THEN $W_{k}=$ Value $_{k}$ (Figure 3-b), the Hamachersum aggregator operator is used to get an aggregated area of waste. Finally, defuzzification transforms this area to a crisp value, which is considered to be the estimation of the waste relative to the input incident. For example: $I N C 1: \mu(x)=$ $0.188 /$ High $+0.718 /$ Medium $+0.000 /$ Low $\Rightarrow$
$Y=1.218$, where $Y$ represents a level of waste as crisp value (Figure $3-b)$.

\subsection{Experimental Setup and Data Incident Organization}

In order to organize the steps of the proposed approach, the following tool was used to collect and process data to incident management: Jira software was used to track all the life cycles of incidents, from identification to closure, thus, to recover the values of the input parameters for the fuzzy process. Each incident will be qualified by an IT team with a crisp value related to the incident level. Hence, what influences all treatment chains supports waste generation. This chain consists of seven key stages of incident response, namely, detect the incident, set up team communication channels, assess the impact and apply a severity level, communicate with customers, escalate to the right responders, delegate incident response roles, resolve the incident. These proceedings are likely to generate wastes such as rework, delays and other more technical stages related to batch and inter-application flow.

\subsection{Algorithm or Steps of Fuzzy Logic Experimentation}

This section represents an iteration of the logical fuzzy application in a real IT service context,

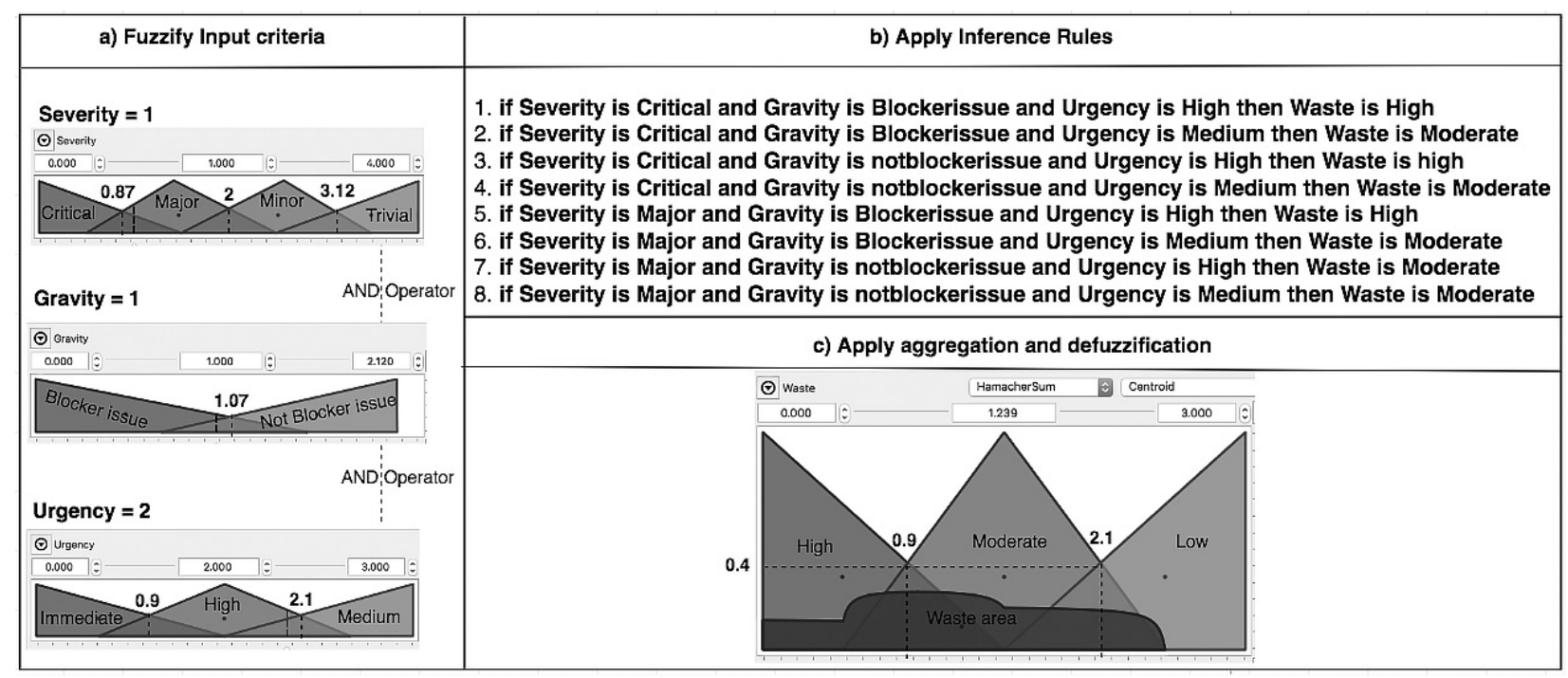

Figure 3. Example of fuzzy logic method application to an input incident. 
especially in IT operation. The FuzzyLite Software was used to fuzzification and defuzzification treatments moreover, fuzzy rules integrations. This tool allows us to visualize the fuzzy approach application, to the effect that Lean improvement processes give high importance to visualization. For each incident entered, a set of rules are activated in order to enable computer aggregations using the humacherSum function and display the potential waste by using the centroid function.

The following steps, laid out in Java language, describe an incident fuzzy process between determination of variables and provision of the net value representing potential waste:

Step 0: Declaration of Variables:

InputVariable $C_{i}=$ new InputVariable();

OutputVariable $W_{i}=$ new

OutputVariable();

Step 1: Declaration the range of value:

Input Value $_{i k}$.setRange(min, max);

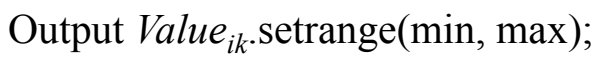

Step 2: Fuzzification Triangular membership function:

using Equation (1)

Step 3: Activation of rules block $\left(R_{k}\right)$ :

RuleBlock $R_{k}=$ new $\operatorname{RuleBlock}() ;$

$R_{k}$.setActivation (new General());

Step 4: Implication and Aggregation of rules:

$R_{k}$ setConjunction(new AlgebraicProd$\operatorname{uct}())$;

$R_{k}$.setDisjunction(new HamacherSum());

$R_{k}$.setImplication(new HamacherProduct());

$W_{i}$.setAggregation(new HamacherSum());

Step 5: display of waste value using centroid defuzzification:

$W_{i}$.setDefuzzifier(new Centroid(100));

This crisp value represents a level of potential waste towards IT incident management process activities. This level will be compared by the actual waste observed at the closure of the incident.

\subsection{Results and Discussion}

Table 4. summarizes the results of the execution of our fuzzy logic approach to twenty input incidents, which produces the waste degree estimation in the output. For each incident, input criteria are evaluated and entered into the system. The corresponding fuzzy set for each incident is highlighted in the "Waste fuzzy set" column. The final result of the fuzzy logic approach is depicted in the "Estimated Waste" column, which corresponds to a crisp value of the waste. The corresponding degree of this estimated value is represented by the "Estimated waste" degree column. It is noteworthy that this estimated degree corresponds to the biggest obtained degree in waste fuzzy set, as described by Equation 1, for each incident. The last column shows the "Observed waste" degree in the deployment according to the experts who were in charge of handling the incidents. This degree was fed by the selfsame experts who have settled the waste linguistic variables and the fuzzy rules.

Several observations could be highlighted from Table 4. Firstly, the observed waste degree is greater than the estimated wastes for almost all the incidents. This reveals that, in general, the actual incident management process is more complex than initially estimated. This may be due to additionally required information or to supplemental tasks essential for the treatment process. This can also result from the experience limitations of stakeholders, which drives the treatment process inefficiently once it is identified and launched.

Further, although both the incident INC18 severity and the estimated waste degree were low, the observed degree waste was high in the experiment. This is explained by the fact that this incident was treated twice. Actually, the affectation of the incident was bad due to the incorrect evaluation and classification at the upstream of its treatment process. This situation is generally known in ITIL as the escalation activity.

Finally, we consider our fuzzy logic based framework to be useful in several contexts. For 
instance, in order to provide a better service quality and respect the Service Level Agreement (SLA), the service IT manager could be able to revise these clauses precisely, by using the estimations provided by the framework. This will bring real intervention time for holding the incident and the SLA clauses closer.

At the same time, by leading this analysis for a long period, and by linking each incident to the item configuration where it was produced, the obtained estimations could be useful in order to enrich a specific item configuration system, called configuration management database (CMDB) as recommended by ITIL [9]. In this way, a set of mechanisms could be applied to enhance the incident management process by a more precise identification of any inconsistences within this database and then ensuring better understanding of the incidents and better estimation of potential wastes.

\section{Conclusion}

Due to unnecessary movement of material and information, excess work-in-process such as queues and pending request, and to unnecessary movement of people in service areas, significant waste could be generated in IT services. In this article, we have provided a fuzzy logic approach, which aims at estimating the generated waste in a given IT service. As the approach allows quantifying this waste based on objective and certain criteria instead of laying out an expert analysis for each waste, we show how the approach could be useful for improving the IT service performance and its SLA clauses.

As a proof of the concept, we have led the approach in the specific context of incident process management as an example of IT service. We worked on a real world case study with twenty incidents and checked the adequacy of our estimation according to the observed waste. As a perspective of this work, we aim at enriching the CMDB database with the knowledge obtained from the historical data in order to optimize the incident management.

\section{References}

[1] N. Ahmad and Z. M. Shamsudin, "Systematic Approach to Successful Implementation of ITIL", Procedia Computer Science, pp. 237-244, 2013. https://dx.doi.org/10.1016/j.procs.2013.05.032

[2] R. Esteves and P. Paulo Alves, "Implementation of an Information Technology Infrastructure Library Process - The Resistance to Change", Procedia Technology, pp. 505-510, 2013. https://dx.doi.org/10.1016/j.protcy.2013.12.056

[3] D. Cannon and D. Wheeldon, "ITIL Service Operation", 1st ed., Office Of Government Commerce (OGC), TSO, 2007.

[4] F. Filardi et al., "Implementation Analysis of Lean Sigma in IT Applications. A Multinational Company Experience in Brazil", Procedia Computer Science, vol. 55, pp. 1221-1230, 2015. https://dx.doi.org/10.1016/j.procs.2015.07.128

[5] F. Pakdil and K. Moustafa Leonard, "Criteria for a Lean Organisation: Development of a Lean Assessment Tool", International Journal of Production Research, vol. 52, no. 15, pp. 4587-4607, 2014.

https://dx.doi.org/10.1080/00207543.2013.879614

[6] T. Welo and G. Ringen, "Beyond Waste Elimination: Assessing Lean Practices in Product Development", Procedia CIRP, vol. 50, pp. 179-185, 2016.

https://dx.doi.org/10.1016/j.procir.2016.05.093

[7] O. Oleghe and K. Salonitis, "Improving the Efficacy of the Lean Index Through the Quantification of Qualitative Lean Metrics", Procedia CIRP, vol. 37, pp. 42-47, 2015.

https://dx.doi.org/10.1016/j.procir.2015.08.079

[8] B. J. Hicks, "Lean Information Management: Understanding and Eliminating Waste", International Journal of Information Management, vol. 27, pp. 233-249, 2007.

https://dx.doi.org/10.1016/j.ijinfomgt.2006.12.001

[9] G. Britain, "ITIL Lifecycle Publication Suite", The Stationery Office (TSO), 2011.

[10] W. Berrahal and R. Marghoubi, "Lean Continuous Improvement to Information Technology Service Management Implementation", in Proc. of the 2nd International Conference on Information Technology for Organizations Development, 2016, pp. 63-64.

https://doi.org/10.1109/IT4OD.2016.7479279

[11] S. Honamore and S. K. Rath, "A Web Service Reliability Prediction Using HMM and Fuzzy Logic Models", Procedia Computer Science, vol. 93, pp. 886-892, 2016.

https://dx.doi.org/10.1016/j.procs.2016.07.273

[12] A. Susilawati et al., "Fuzzy Logic Based Method to Measure Degree of Lean Activity in Manufac- 
turing Industry", Journal of Manufacturing Systems, vol. 34, pp. 1-11, 2015.

https://dx.doi.org/10.1016/j.jmsy.2014.09.007

[13] G. J. Klir and B. Yuan, "Fuzzy Sets and Fuzzy Logic", Prentice Hall, 1995.

[14] H. Williams and R. Duray, "Making IT Lean. Applying Lean Practices to the Work of IT", Taylor Francis Group, LLC International Standard Book Number-13: 978-1-4398-7603-9 (eBook), 2013.

[15] R. Asnan et al., "Managing Change on Lean Implementation in Service Sector", Procedia-Social and Behavioral Sciences, vol. 211, pp. 313-319, 2015.

https://dx.doi.org/10.1016/j.sbspro.2015.11.040

[16] G. Spalding and G. Case, "ITIL Service Continuous Improvement", 1st ed., Office of Government Commerce (OGC), TSO, 2007.

[17] F. Farid et al., "A Fuzzy Logic Approach for Quality of Service Quantification in Wireless and Mobile Networks", 10th IEEE International Workshop on Performance and Management of Wireless and Mobile Networks, Edmonton, Canada, 2014

https://dx.doi.org/10.1109/LCNW.2014.6927713

[18] M. Rother, "Toyota Kata: Managing People for Improvement, Adaptiveness and Superior Results", 1st ed., McGraw-Hill Education, 2010.

[19] B. R. Staats et al., "Lean Principles, Learning, and Knowledge Work: Evidence from a Software Services Provider", Journal of Operations Management, vol. 29, pp. 376-390, 2011. https://dx.doi.org/10.1016/j.jom.2010.11.005

[20] Y.-M. Wang, "Centroid Defuzzification and the Maximizing Set and Minimizing Set Ranking Based on Alpha Level Sets", Computers \& Industrial Engineering, vol. 57, issue 1, pp. 228-236, 2009.

https://dx.doi.org/10.1016/j.cie.2008.11.014

[21] A. Talon and C. Curt, "Selection of Appropriate Defuzzification Methods: Application to the Assessment of Dam Performance", Expert Systems with Applications, vol. 70, pp.160-174, 2017. https://dx.doi.org/10.1016/j.eswa.2016.09.004

[22] G. Beliakov and J. Warren, "Appropriate Choice of Aggregation Operators in Fuzzy Decision Support Systems", IEEE Transactions on Fuzzy Systems, vol. 9, pp. 773-784, 2001. https://dx.doi.org/10.1109/91.971696

[23] W. V. Leekwijck and E. E. Kerre, "Defuzzification: Criteria and Classification", Fuzzy Sets and Systems, vol. 108, no. 2, pp. 159-178, 1999. https://dx.doi.org/10.1016/S0165-0114(97)00337-0

[24] M. D. de Barros et al., "Mapping of the Scientific Production on the ITIL Application Published in the National and International Literature", Procedia Computer Science, vol. 55, pp. 102-111, 2015. https://dx.doi.org/10.1016/j.procs.2015.07.013

[25] W. Berrahal et al., "Lean to Identification and Categorization of Wastes in IT Service: Focusing on IT Operation Processes" in Proc. of the Conference ASD: Big Data \& Applications 12th edition of the Conference on Advances of Decisional Systems, 2018, pp. 548-556.

[26] W. Berrahal and R. Marghoubi, "Lean IT Management to Service Operation Processes: Towards an Added Value Without Wastes", in Proc. of the 7th. International Conference SIIE on Information Systems and Economic Intelligence, 2017, pp. 76-81.

[27] D. Adebanjo et al., "Prioritizing Lean Supply Chain Management Initiatives in Healthcare Service Operations: A Fuzzy-AHP Approach", Production Planning \& Control, vol. 27, issue 12, pp. 953-966, 2016.

https://dx.doi.org/10.1080/09537287.2016.1164909

[28] N.V. Hop and N. H. Phuong Nam, "Fuzzy Analytical Hierarchical Process for Hub-Location Selection Using Superior and Inferior Degree and Dominance Measure", 13th International Congress on Logistics and SCM Systems, 2018.

[29] L. Liu et al., "A modified Fuzzy C-Means (FCM) Clustering Algorithm and Its Application on Carbonate Fluid Identification", Journal of Applied Geophysics, vol. 129, pp. 28-35, 2016. https://dx.doi.org/10.1016/j.jappgeo.2016.03.027

[30] H. S. Pannu et al., "Multi-Objective Particle Swarm Optimization-Based Adaptive Neuro-Fuzzy Inference System for Benzene Monitoring", Neural Comput \& Applications, vol. 31, pp. 2195-2205, 2019. https://dx.doi.org/10.1007/s00521-017-3181-7

[31] D. Singh et al., "Single Image Dehazing Using Gradient Channel Prior", Applied Intelligence, vol. 49, pp. 4276-4293, 2019. http://dx.doi.org/10.1007/s10489-019-01504-6

[32] M. Kaur and D. Singh, "Fusion of Medical Images Using Deep Belief Networks", Cluster Computing, 2019. http://dx.doi.org/10.1007/s10586-019-02999-x

[33] M. Kaur et al., "Multi-Objective Differential Evolution Based Random Forest for e-Health Applications", Modern Physics Letters B, vol. 33, no. 5, 2019.

http://dx.doi.org/10.1142/S0217984919500222 
Contact address:

Wadie Berrahal

National postal and telecommunication institute

Rabat

Morocco

e-mail: berahal@inpt-ac.ma

Rabia Marghoubi

National postal and telecommunication institute

Rabat

Morocco

e-mail: m.rabia@inpt.ac.ma

Zineb El Akkaoui

National postal and telecommunication institute

Rabat

Morocco

e-mail: elakkaoui@inpt.ac.ma
Wadie Berrahal is a PhD student in computer science at the National Post and Telecommunications Institute (INTP), Rabat, Morocco. He trained in digital technologies at the EMSI engineering school in 2009. He received his master degree in decision support system (DSS) and project management from the University of Kenitra, Morocco in 2014. He has significant expertise in the computer science industry, 11 years in design and customization of software together with optimizing existing software architectures to improve efficiency or overcome problems. In addition, he performed assessments of software requirements and user needs to determine the feasibility and efficiency of software. He participated in several international conferences and workshops, as well as, trainings and presentations on the software quality, Agile and Lean IT methodologies.

RABIA MARGHOUBI received her PhD degree in Computer Science from the Faculty of Sciences and Techniques of Mohammedia, Hassan II University, Morocco, in 2006, and her Habilitation in Computer Science from the Faculty of Sciences and Techniques of Settat - Hassan I University Morocco in 2013. She is professor of computer science and coordinator of the "Advanced Software Engineering for Digital Services (ASEDS)" program at the National Post and Telecommunications Institute (INPT), Morocco. Her principal research interests are information system management, project management and spatial data mining. Rabia has published in international peer-reviewed conferences and journals. She has also been a reviewer for top-level conferences and journals, and a PC member of international conferences and workshops.

Zineb El AkKaoul is a Professor in the Department of Economics, Management, Languages and Communication at the INPT Engineering School. She obtained her PhD from the University Libre de Bruxelles at the Department of Computer \& Decision Engineering (CoDE) in 2014. She received her master degree in computer science from ENSIAS (Ecole Nationale Supérieure d'Informatique et d'Analyse des Systèmes) of Morocco 2007. Her research interests include decision support systems, machine learning, data-mining, and model-driven development. She has been a co-organizer of the International Conference on Technology Management, Operations and Decisions (IEEE ICTMOD) series, and is a member of program committees of several international conferences and workshops. 